\title{
Dan-Shen-Yin protects the heart against inflammation and oxidative stress induced by acute ischemic myocardial injury in rats
}

\author{
KUI-PO YAN ${ }^{1}$, YONG GUO $^{2}$, ZHIHUA XING $^{1}$, XI HUANG $^{1}$, SHENGPING DAI $^{3}$, \\ MIN DUAN ${ }^{4}$, XINHUA SUN ${ }^{4}$, WEI HUANG ${ }^{1}$ and WEIJUN PENG ${ }^{1}$ \\ ${ }^{1}$ Institute of Integrated Traditional Medicine and Western Medicine; ${ }^{2}$ Department of Neurosurgery, Xiangya Hospital, \\ Central South University, Changsha 410008; ${ }^{3}$ Department of Cardiology, The PLA 152 Central Hospital, \\ Pingdingshan 467000; ${ }^{4}$ Department of Cardiology, The First Affiliated Hospital \\ of Henan College of TCM, Zhengzhou 450008, P.R. China
}

Received September 8, 2011; Accepted November 16, 2011

DOI: $10.3892 /$ etm.2011.404

\begin{abstract}
Dan-Shen-Yin (DSY) is a well-known traditional Chinese formula which is widely used in clinical practice for the treatment of coronary heart disease (CHD) and has produced a favorable effect in China. The present study was designed to examine whether or not acute oral DSY can protect the heart against myocardial infarction in acute myocardial ischemic rats. If so, we would then investigate the anti-inflammatory and anti-oxidant mechanisms involved. The left anterior descending coronary artery was occluded to induce myocardial ischemia in the hearts of Sprague-Dawley rats. At the end of the 3-h ischemic period (or $24 \mathrm{~h}$ for infarction size), we measured the myocardial infarction size, inflammatory factors and the activities of anti-oxidative enzymes. DSY reduced the infarction size and the serum levels of C-reactive protein, interleukin-6, tumour necrosis factor- $\alpha$ and malondialdehyde and increased the activities of superoxide dismutase and the serum levels of glutathione. The results show that DSY exerts significant cardioprotective effects against acute ischemic myocardial injury in rats, possibly through its anti-inflammatory and anti-oxidant properties, and may thus be used as a potential therapeutic reagent for the treatment of CHD.
\end{abstract}

\section{Introduction}

Cardiovascular disease is the leading cause of death worldwide, accounting for more than 900,000 deaths annually in the United States alone (1). Clinical study shows that its main cause

Correspondence to: Dr Zhihua Xing, Institute of Integrated Traditional Medicine and Western Medicine, Xiangya Hospital, Central South University, Changsha 410008, P.R. China

E-mail: ykp19821122@163.com

Key words: traditional Chinese formula, Dan-Shen-Yin, coronary heart disease, inflammation, oxidative stress of death is acute myocardial infarction (AMI) (2). The most common cause of MI is reduced myocardial perfusion due to coronary artery narrowing caused by a thrombus, that develops on a disrupted atherosclerotic plaque. Pathological studies have shown that the most common underlying molecular and cellular pathophysiology of disrupted atherosclerotic plaque is arterial inflammation in MI (3), and also oxidative stress plays a critical role in AMI $(4,5)$. In recent years, pharmacological studies have indicated that many traditional plants and their extracts have anti-inflammatory (6-9) and anti-oxidant $(10,11)$ effects on cardiovascular disease.

Dan-Shen-Yin (DSY) is a well-known traditional Chinese formula comprising Salvia Miltiorrhiza, Sandalwood and Fructus Amomi. It is widely used in clinical practice for the treatment of diabetes $(12,13)$ and coronary heart disease (CHD) $(14,15)$ and has produced a favorable effect (12-15).

However, the anti-inflammatory and anti-oxidant mechanisms of DSY are unclear. The object of the present study was to evaluate whether or not acute oral DSY can protect the heart against AMI rats. If so, we would then investigate the anti-inflammatory and anti-oxidant mechanisms involved.

\section{Materials and methods}

Preparation of DSY decoction. The crude drugs of DSY, including $120 \mathrm{~g}$ of Salvia Miltiorrhiza, $18 \mathrm{~g}$ of Sandalwood and $18 \mathrm{~g}$ of Fructus Amomi, were purchased from LBX Pharmacy (Zhengzhou, China). To keep the consistency of the herbal chemical ingredients, all the herbal components were originally obtained from the standard native sources, as stated above, with GAP grade, and the drugs were extracted with standard methods according to the Chinese Pharmacopeoia (China Pharmacopoeia and Committee, 2000). Voucher specimens (No. 100322) had been kept. The mixture of DSY was produced by boiling with distilled water at $100^{\circ} \mathrm{C}$ for $30 \mathrm{~min}$ twice, and the drug solution was vacuum cool-dried, rendered into drug powder and dissolved with distilled water, with a final concentration of $2.5 \mathrm{~g} / \mathrm{ml}$ (equivalent to dry weight of raw materials). 
Experimental protocol. Male Sprague-Dawley rats $(n=40)$ weighing 180-220 g were purchased from the Henan Laboratory Animal Center (Henan, Zhengzhou, China). Rats were housed in temperature-controlled $\left(22 \pm 2^{\circ} \mathrm{C}\right)$ and humidity-controlled $(55 \pm 5 \%)$ rooms under 12 -h light-dark cycles. Rats had free access to solid rodent chow and tap water. Animals were allowed a 1-week acclimatization period prior to entry into any experimental protocol. The animal experiments were conducted in accordance with the Use of Laboratory Animals by the US National Institutes of Health (1996). The rat dose of DSY in the present study was converted according to the principles described below. The low dosage of DSY used in rats was $5 \mathrm{~g} / \mathrm{kg}$, that is, the dose used was 6.7 times the dose used in humans. This is consistent with a previous study (16). The high dose used in rats was $10 \mathrm{~g} / \mathrm{kg}$, which is equivalent to the dose administered to clinical patients without causing significant side-effects. The experiments were performed $30 \mathrm{~min}$ after the administration of DSY (5 or $10 \mathrm{~g} / \mathrm{kg}$ ) or $0.9 \% \mathrm{NaCl} 20 \mathrm{ml} / \mathrm{kg}$.

Surgical preparation of animals. We performed the surgical procedure according to a previous study (17), with some modifications. In brief, the rats were anesthetized intraperitoneally with pentobarbitone at a dose of $36 \mathrm{mg} / \mathrm{kg}$ and subcutaneous peripheral limb electrodes were inserted and the ECG was recorded for the entire duration of the experiment (AD Instruments, Australia). After performing a left thoracotomy, the incised area was extended using forceps and the pericardium was opened. The heart was then pushed out of the chest and the left anterior descending coronary artery was ligated using a 3/0 silk thread. Successful ligation was verified by the occurrence of arrhythmias and, visually, by the color change of the ischemic area. The heart was immediately returned to its anatomical position and the chest was closed, while slight pressure was applied from the outside, so that air did not remain in the chest. The skin was then sutured. Forty male Sprague-Dawley rats (180-220 g) were randomly assigned to four groups: i) sham-operated control group (sham MI), rats underwent the same surgical procedures except that the suture passing under the coronary artery was not tied; ii) vehicle control group (MI + vehicle), rats were orally administered the vehicle (0.9\% NaCl, $20 \mathrm{ml} / \mathrm{kg}$ ); iii) low-dose DSY group (MI + DSY), rats were orally administered DSY extract $(5 \mathrm{~g} / \mathrm{kg}) 30 \mathrm{~min}$ prior to ischemia induction; iv) high-dose DSY group (MI + DSY), rats were orally administered DSY extract $(10 \mathrm{~g} / \mathrm{kg})$ $30 \mathrm{~min}$ prior to ischemia induction. At the end of the 3 -h ischemic period (or $24 \mathrm{~h}$ for infarct size), the heart was quickly excised and the cardiac tissue was processed according to the procedures described below; the mortality rate was $\sim 5 \%$.

Determination of the infarction size. The determination of the infarction size was performed as previously described $(18,19)$. We injected $1 \mathrm{ml} \mathrm{2 \%}$ Evans blue dye into the left ventricular cavity $24 \mathrm{~h}$ after MI induction. The normally perfused region was stained blue and the area at risk (Ar) was outlined. The heart was then removed, frozen at $-20^{\circ} \mathrm{C}$ and sliced into $1-\mathrm{mm}$ thick transverse sections. The sections were incubated for $15 \mathrm{~min}$ at $37^{\circ} \mathrm{C}$ in a $1 \%$ solution of triphenyltetrazolium chloride (TTC) (SCR, Shanghai, China) to differentiate necrotic (pale) from non-necrotic Ar. The Ar as a percentage of the left ventricle $(\mathrm{Lv})(\mathrm{Ar} / \mathrm{Lv})$, and the area of necrosis (An) as a percentage of the Ar (An/Ar) were calculated using Image Pro Plus 6.0 software.

Inflammatory factors assay. For the assays of the serum levels of C-reactive protein (CRP), interleukin-6 (IL-6) and tumour necrosis factor- $\alpha$ (TNF- $\alpha$ ), a commercial ELISA kit (Uscnlife, Zhengzhou, Henan, China) was used according to the recommended protocol. At the end of the 3 -h ischemic period, we used a serum separator tube and allowed whole blood to clot for $30 \mathrm{~min}$ before centrifugation for $15 \mathrm{~min}$ at $1,000 \mathrm{x} \mathrm{g}$. We then removed the serum. According to the kit instructions, $100 \mu \mathrm{l}$ of standard, blank or sample were added per well and incubated for $2 \mathrm{~h}$ at $37^{\circ} \mathrm{C}$, after which the liquid was removed. Detection reagent $\mathrm{A}(100 \mu \mathrm{l})$ was added to each well, incubated for $1 \mathrm{~h}$ at $37^{\circ} \mathrm{C}$, and we used wash buffer to wash each well. We then added $100 \mu \mathrm{l}$ of detection reagent $\mathrm{B}$ to each well, incubated for $1 \mathrm{~h}$ at $37^{\circ} \mathrm{C}$, and washed each well again. After adding $90 \mu \mathrm{l}$ of substrate solution, followed by incubation for $20 \mathrm{~min}, 50 \mu \mathrm{l}$ of stop solution were added at room temperature. The optical density of each well was determined using a microplate spectrophotometer (BioTek, Winooski, VT, USA) at a $450 \mathrm{~nm}$ wavelength. Finally, we created a standard curve to interpret the results.

Antioxidant assay. For the assays of superoxide dismutase (SOD), glutathione (GSH) and malondialdehyde (MDA) activities, blood was sampled from the abdominal aorta and serum was obtained after centrifugation at 3,000 rpm for $10 \mathrm{~min}$. We used diagnostic kits (Jiancheng, Nanjing, China) to determine the levels of SOD, MDA and GSH according to the manufacturer's instructions.

Statistical analysis. All data were expressed as the means \pm standard deviation (SD). A Dunnett's t-test was used to compare the data obtained before and after occlusion. In the animal study, one-way ANOVA was used to analyse differences in parameters. A probability of $<0.05$ was considered to be statistically significant.

\section{Results}

Effect of DSY on infarction size. Images of MI size in the sham-, vehicle- and DSY-treated groups are shown in Fig. 1. The An (white area), the Ar (red staining and white area) and the area of the Lv were measured using Image Pro Plus 6.0 software. The effects of DSY on Ar/Lv and An/Ar are shown in Fig. 1. There was no significant difference in Ar/Lv among the groups. The administration of $5 \mathrm{~g} / \mathrm{kg}$ DSY significantly reduced the An/Ar compared to the vehicle control group (vs. $49.85 \pm 9.13 \%, \mathrm{P}<0.01 ; 25.53 \pm 11.13$ vs. $49.85 \pm 9.13 \%, \mathrm{P}<0.01)$. The administration of $10 \mathrm{~g} / \mathrm{kg}$ DSY significantly reduced the An/Ar compared to the DSY $5 \mathrm{~g} / \mathrm{kg}$ group (25.53 $\pm 11.13 \mathrm{vs}$. $29.86 \pm 6.27 \%, \mathrm{P}<0.05$ )

Effect of DSY on the serum levels of CRP, TNF- $\alpha$ and IL-6. To determine if DSY has an anti-inflammatory effect, the serum levels of CRP, TNF- $\alpha$ and IL- 6 were determined by ELISA. As shown in Fig. 2A-C, coronary occlusion significantly increased the serum levels of CRP, TNF- $\alpha$ and IL- 6 compared to the sham group (all $\mathrm{P}<0.01$ ). As shown in Fig. $2 \mathrm{~A}$ and $\mathrm{C}$, the 


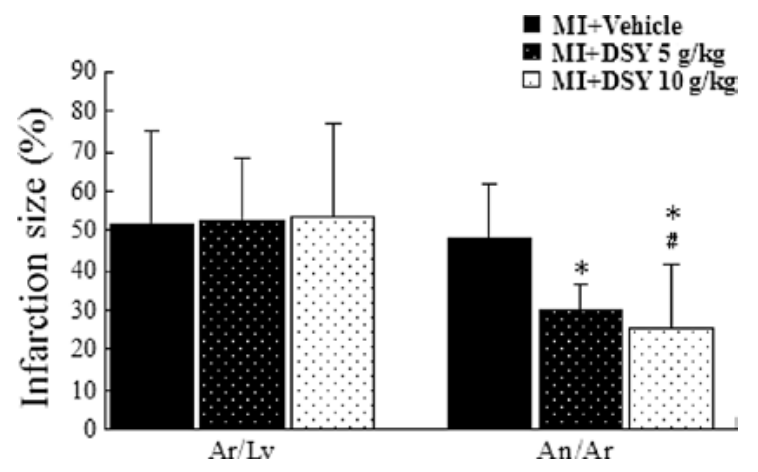

Figure 1. Effect of DSY on the size of myocardial infarction. Ar was expressed as a percentage of $\mathrm{Lv}(\mathrm{Ar} / \mathrm{Lv})$ and $\mathrm{An}$ was expressed as a percentage of $\mathrm{Ar}$ (An/Ar). ${ }^{*} \mathrm{P}<0.05$ vs. $\mathrm{MI}+$ vehicle $(\mathrm{n}=8) .{ }^{.} \mathrm{P}<0.05$ vs. MI + DSY $5 \mathrm{~g} / \mathrm{kg}(\mathrm{n}=10)$. MI, myocardial infarction; DSY, Dan-Shen-Yin.

\section{A}

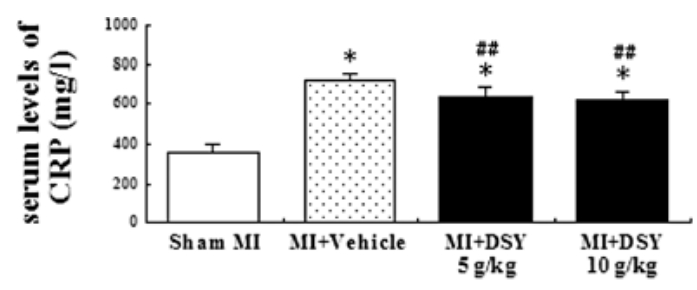

B
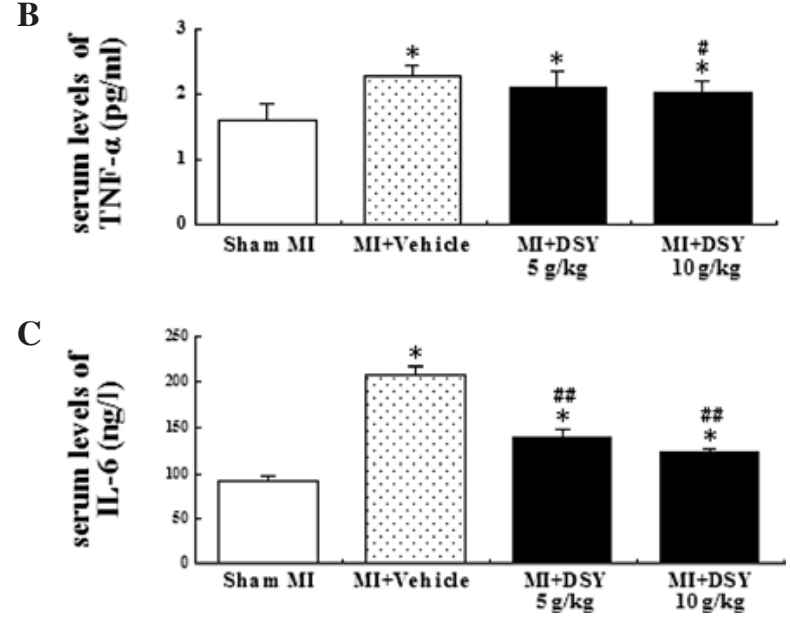

Figure 2. Effects of DSY on the concentrations of circulating inflammatory markers C-reactive protein (CRP), tumour necrosis factor- $\alpha$ (TNF- $\alpha$ ), serum fructosamine and interleukin-6 (IL-6) in sham-, vehicle- and DSY-treated groups. (A) Serum CRP levels; (B) serum TNF- $\alpha$ levels; (C) serum IL-6 levels in rats. Serum levels of TNF- $\alpha$, IL- 6 and CRP were determined by ELISA. ${ }^{*} \mathrm{P}<0.01$ vs. the sham group $(\mathrm{n}=10) ;{ }^{*} \mathrm{P}<0.05$ and ${ }^{\# \#} \mathrm{P}<0.01$ vs. the vehicle group $(\mathrm{n}=8)$. MI, myocardial infarction; DSY, Dan-Shen-Yin.

serum levels of CRP and IL-6 in the DSY-treated group were significantly lower than those in the vehicle group $(\mathrm{P}<0.01)$. The serum levels of TNF- $\alpha$ in the DSY $(10 \mathrm{~g} / \mathrm{kg})$-treated group were significantly lower compared to those in the vehicle group $(\mathrm{P}<0.05)$; there was no significant difference between $5 \mathrm{~g} / \mathrm{kg}$ DSY and the vehicle groups $(\mathrm{P}>0.05)$. However, there were no significant differences in the serum levels of CRP, TNF- $\alpha$ and IL- 6 between the 10 and $5 \mathrm{~g} / \mathrm{kg}$ DSY groups $(\mathrm{P}>0.05)$.

Anti-oxidant assay. The serum levels of SOD and GSH in the DSY-treated groups were significantly higher $(\mathrm{P}<0.01$
A

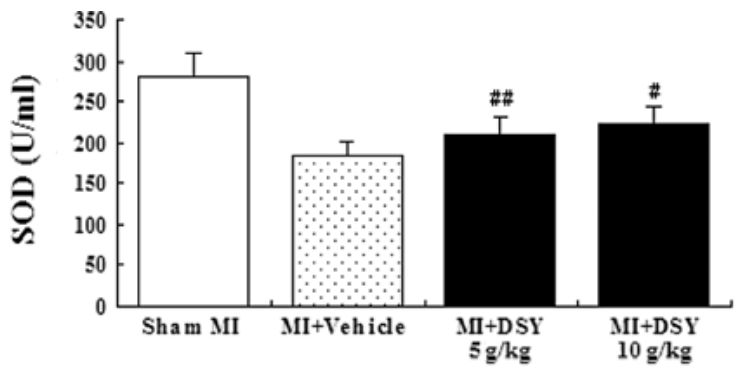

B
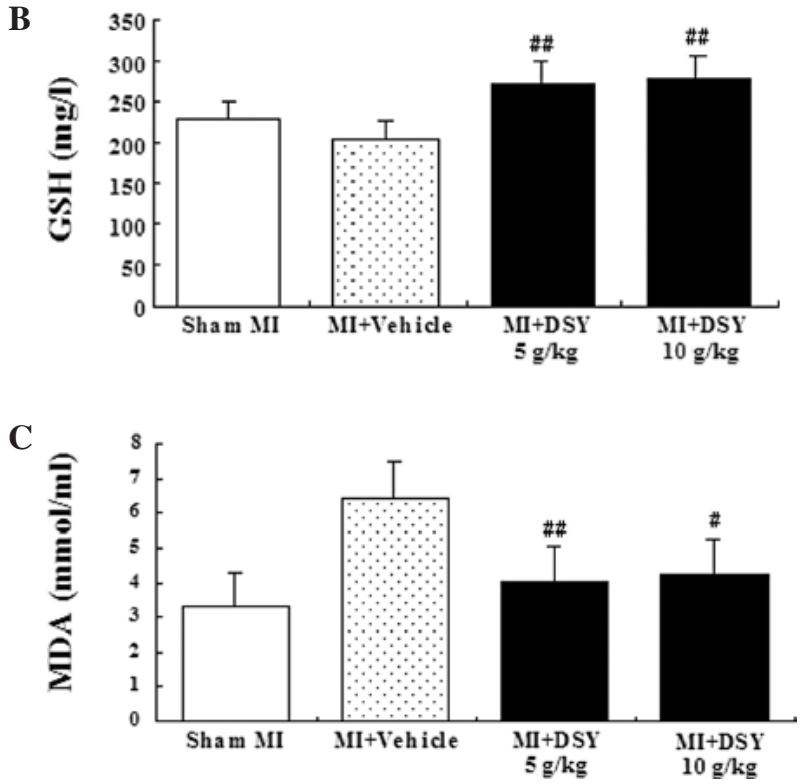

Figure 3. Antioxidant assay and DSY effects. (A) Superoxide dismutase (SOD); (B) glutathione (GSH); (C) malondialdehyde (MDA). Sham MI group $(\mathrm{n}=10), \mathrm{MI}+$ vehicle group (orally administered the vehicle, $\mathrm{n}=8), \mathrm{MI}+\mathrm{DSY}$ (orally administered DSY extract $5 \mathrm{~g} / \mathrm{kg} ; \mathrm{n}=10$ ) and MI + DSY (orally administered DSY extract $10 \mathrm{~g} / \mathrm{kg} ; \mathrm{n}=10) .{ }^{\#} \mathrm{P}<0.05$ vs. the $\mathrm{MI}+$ vehicle group $(\mathrm{n}=8)$; ${ }^{\# \#} \mathrm{P}<0.01$ vs. the $\mathrm{MI}+$ vehicle group $(\mathrm{n}=8)$

or 0.05 ; Fig. $3 \mathrm{~A}$ and $\mathrm{B}$ ), while the serum MDA levels in the DSY-treated groups were significantly lower compared to the vehicle groups $(\mathrm{P}<0.01$ or 0.05 ; Fig. $3 \mathrm{C})$. There were also significant differences between the DSY-treated groups and sham groups in the serum levels of SOD, GSH and MDA $(\mathrm{P}<0.01$ or 0.05$)$. In addition, there were no significant differences between the $5 \mathrm{~g} / \mathrm{kg}$ DSY-treated group and the $10 \mathrm{~g} / \mathrm{kg}$ DSY-treated group in the serum levels of SOD, GSH and MDA (all P>0.05)

\section{Discussion}

In this study, 2 out of 10 rats in the vehicle group died, while in the DSY-treated groups all survived. This is the most direct evidence that AMI has a high mortality risk and DSY has a protective role. Notably, we found that DSY can reduce infarction size (Fig. 1). Infarction size is a major determinant of mortality in AMI (20) and an important determinant of the prognosis of MI. Therefore, the limitation of the infarction size must be considered a major therapeutic goal. Numerous investigations have focused on targeting and exploring several pharmacological agents in an attempt to reduce experimental 
MI size (21). Over the years, Chinese medicinal herbs and their extracts have received great attention $(22,23)$. Further studies have found that DSY decreases the concentrations of CRP, TNF- $\alpha$ and IL- 6 and reduces the amounts of MDA, but enhances SOD and GSH activities in AMI rats.

CRP is a prototypic acute-phase protein produced in the liver in response to inflammatory signals (24). Evidence has linked CRP to risk of cardiovascular events, a reflection of the involvement of inflammation in atherosclerosis and its complications $(25,26)$. CRP is a sensitive, non-specific systemic marker of inflammation (27), and strong evidence indicates that CRP is associated with CHD events (28). CRP concentrations in the acute phase have been suggested to reflect pre-existing coronary plaque instability associated with the onset of AMI (29). Also, CRP has been known for more than 25 years to bind to LDL $(30,31)$, and it has been detected in atherosclerotic plaques (32). Laboratory and clinical evidence has demonstrated that CRP has been examined as a surrogate marker of other inflammatory mediators, such as IL- 6 and TNF- $\alpha$, to better understand the inflammatory component of atherosclerosis (33). TNF- $\alpha$ and IL-6 are antigen non-specific glycoproteins that are synthesized rapidly and released locally by immune cells in response to injury (34). Additionally, they are pro-inflammatory cytokines with crucial roles in cardiomyocyte apoptosis (35). Cardiomyocytes express functional TNF type-1 receptors and have been shown to undergo apoptosis after stimulation with TNF- $\alpha$ in vitro (36). In the present study, we found significantly reduced levels of CRP, TNF- $\alpha$ and IL- 6 in the DSY-treated groups compared to the vehicle groups after AMI induction. Thus, DSY, through its anti-inflammatory properties, protects the cardiomyocytes from the effects of AMI.

Oxidative stress is the leading cause of the worst outcome of MI (37). Oxidative stress is increased in the myocardium following infarction, playing significant roles in cardiac myocyte death and loss of cardiac function (38). SOD is a very important enzyme and its activity reflects the cellular capability of scavenging/quenching free radicals (39). MDA, the degradation product of the oxygen-derived free radicals and lipid oxidation, interferes with the metabolism of proteins, glucose and nucleic acid, which results in a decrease in enzyme activity, template dysfunction of nucleic acid and injury of tissues and cells (39). The levels of MDA are then regarded as the degree of lipid peroxidation. In view of evaluating the basal metabolism of free radicals, the activity of SOD and the levels of MDA are the principal pathophysiological parameters. GSH, being an important cellular reductant, is involved in the protection against free radicals, peroxides and toxic compounds (40). Depletion of GSH is one of the primary factors that permit lipid peroxidation (41). DSY reduced the serum levels of MDA, increased the activities of SOD and the serum levels of GSH. DSY increased the activities of anti-oxidant defense enzymes, including SOD and GSH, and reduced MDA in the serum levels of AMI rats in this study. These results indicate that DSY scavenges various free radicals effectively through enhancing the activities of the anti-oxidant enzymes in AMI rats. Furthermore, the antioxidant enzymes in the DSY-treated groups were found to be enhanced by DSY.

This study, thus, shows that DSY reduces inflammatory factors and scavenges free radicals through enhancing anti- oxidant defense enzymes, and reduces free radical production. These could be major factors contributing to the cardioprotective effects of DSY.

In conclusion, our results suggest that Dan-Shen-Yin has beneficial effects on oxidative stress and inflammation that are related to the infarction size in AMI rats, and may thus produce a higher survival rate. Thus, it is valuable to develop this formula into a potential therapeutic reagent according to modern pharmacological standards for use in the treatment of cardiovascular disease.

\section{References}

1. Razzouk L, Mathew V, Lennon RJ, et al: Aspirin use is associated with an improved long-term survival in an unselected population presenting with unstable angina. Clin Cardiol 33: 553-558, 2010.

2. Kloner RA: Natural and unnatural triggers of myocardial infarction. Prog Cardiovasc Dis 48: 285-300, 2006.

3. Anderson JL, Adams CD, Antman EM, et al: ACC/AHA 2007 Guidelines for the Management of Patients with Unstable Angina/ Non-ST-Elevation Myocardial Infarction: a report of the American College of Cardiology/American Heart Association Task Force on Practice Guidelines (Writing Committee to Revise the 2002 Guidelines for the Management of Patients with Unstable Angina/ Non-ST-Elevation Myocardial Infarction): developed in collaboration with the American College of Emergency Physicians, the Society for Cardiovascular Angiography and Interventions, and the Society of Thoracic Surgeons: endorsed by the American Association of Cardiovascular and Pulmonary Rehabilitation and the Society for Academic Emergency Medicine. Circulation 116: e148-e304, 2007.

4. Ueda S, Yamagishi S, Matsui T, et al: Administration of pigment epithelium-derived factor inhibits left ventricular remodeling and improves cardiac function in rats with acute myocardial infarction. Am J Pathol 2: 591-598, 2011.

5. Misra MK, Sarwat M, Bhakuni P, et al: Oxidative stress and ischemic myocardial syndromes. Med Sci Monit 15: RA209-RA219, 2009.

6. Zhang $\mathrm{H}$, Wang WR, Lin R, et al: Buyang Huanwu decoction ameliorates coronary heart disease with Qi deficiency and blood stasis syndrome by reducing CRP and CD40 in rats. J Ethnopharmacol 130: 98-102, 2010.

7. Yin HQ, Wang B, Zhang JD, et al: Effect of traditional Chinese medicine Shu-Mai-Tang on attenuating TNF $\alpha$-induced myocardial fibrosis in myocardial ischemia rats. J Ethnopharmacol 188: 133-139, 2008.

8. Liu Y, Lin R, Shi XL, et al: The roles of buyang huanwu decoction in anti-inflammation, antioxidation and regulation of lipid metabolism in rats with myocardial ischemia. Evid Based Complement Alternat Med: March 10, 2011 (E-pub ahead of print).

9. Asli CI, Rochelle F, Loren W, et al: Herbal and traditional Chinese medicine for the treatment of cardiovascular complications in diabetes mellitus. Curr Diabetes Rev 4: 320-328, 2008.

10. Ibarra-Alvarado C, Rojas A, Mendoza S, et al: Vasoactive and antioxidant activities of plants used in Mexican traditional medicine for the treatment of cardiovascular diseases. Pharm Biol 7: 732-739, 2010.

11. Chon SU,Heo BG, Park YS, et al: Total phenolics level, antioxidant activities and cytotoxicity of young sprouts of some traditional Korean salad plants. Plant Foods Hum Nutr 1: 25-31, 2009.

12. Huang HQ: Dan-Shen-Yin and Er-Chen-Tang treat diabetic gastroparesis 40 cases. J New Chinese Med 39: 68, 2007.

13. Hu MF: Jian-Pi-Wan and Dan-Shen-Yin treat diabetic gastroparesis 60 cases. Chin J Tradit Med Sci Tech 16: 150-151, 2009.

14. Xie M: Sheng-Mai-San and Dan-Shen-Yin treat coronary heart disease 60 cases. Chin Med Mod Dist Educ Chin 6: 1357, 2008.

15. Liu RS: Jia-Wei-Dan-Shen-Yin treat coronary heart disease 68 cases. Chin J Integr Med on Cardio-/Cerebrovascular Disease 8: 604, 2010.

16. Pinkel D: The use of body surface area as a criterion of drug dosage in cancer chemotherapy. Cancer Res 18: 853-856, 1958.

17. Qin F, Liu YX,Zhao HW, et al: Chinese medicinal formula GuanXin-Er-Hao protects the heart against oxidative stress induced by acute ischemic myocardial injury in rats. Phytomedicine 16: 215-221, 2009 . 
18. Zhao HW, Qin F, Liu YX, et al: Antiapoptotic mechanisms of Chinese medicine formula, Guan-Xin-Er-Hao, in the rat ischemic heart. Tohoku J Exp Med 216: 309-316, 2008.

19. Zhao J, Huang X, Tang W, et al: Effect of oriental herbal prescription Guan-Xin-Er-Hao on coronary flow in healthy volunteers and anti-apoptosis on myocardial ischemia-reperfusion in rat models. Phytother Res 21: 926-931, 2007.

20. Thiele H, Hildebrand L, Schirdewahn C, et al: Impact of high-dose N-Acetylcysteine versus placebo on contrast-induced nephropathy and myocardial reperfusion injury in unselected patients with ST-segment elevation myocardial infarction undergoing primary percutaneous coronary intervention. The LIPSIA-N-ACC (Prospective, Single-Blind, Placebo-Controlled, Randomized Leipzig Immediate PercutaneouS Coronary Intervention Acute Myocardial Infarction N-ACC) Trial. J Am Coll Cardiol 55: 2201-2209, 2010.

21. Ling HY and Lou YJ: Total flavones from Elsholtzia blanda reduce infarct size during acute myocardial ischemia by inhibiting myocardial apoptosis in rats. J Ethnopharmacol 101: 169-175, 2005.

22. Zhou JY, Fan Y, Kong JL, Wu DZ and Hu ZB: Effect of components isolated from Astragalus membranaceus Bunge on cardiac function injured by myocardial ischemia reperfusion in rats. Zhongguo Zhong Yao Za Zhi 25: 300-302, 2000.

23. Qi BL and Cheng B: Effect of the daidzein on protect of cardiovascular in unsexed rats. J Chin Clin Med 3: 18-19, 2002

24. Agrawal A: CRP after 2004. Mol Immunol 42: 927-930, 2005.

25. Hansson GK and Libby P: The immune response in atherosclerosis: a double-edged sword. Nat Rev Immunol 6: 508-519, 2006

26. Libby P, Willerson JT and Braunwald E: C-reactive protein and coronary heart disease. N Engl J Med 351: 295-298, 2004.

27. Pepys MB and Hirschfield GM: C-reactive protein: a critical update. J Clin Invest 111: 1805-1812, 2003.

28. Buckley DI, Fu RW, Freeman M, et al: C-reactive protein as a risk factor for coronary heart disease: a systematic review and metaanalyses for the U.S. preventive services task force. Ann Intern Med 151: 483-495, 2009.

29. Kojima S, Funahashi T, Sakamoto T, et al: The variation of plasma concentrations of a novel, adipocyte derived protein, adiponectin, in patients with acute myocardial infarction. Heart 89: 667-668, 2003.
30. Beer F, Soutar AK, Baltz ML, et al: Low density and very low density lipoproteins are selectively bound by aggregated C-reactive protein. J Exp Med 156: 230-242, 1982.

31. Pepys MB, Rowe IF and Baltz ML: C-reactive protein: binding to lipids and lipoproteins. Int Rev Exp Pathol 27: 83-111, 1985.

32. Zhang YX, Cliff WJ, Schoefl GI, et al: Coronary C-reactive protein distribution: its relation to development of atherosclerosis. Atherosclerosis 145: 375-379, 1999.

33. Rifai N and Ridker PM: High-sensitivity C-reactive protein: a novel and promising marker of coronary heart disease. Clin Chem 47: 403-411, 2001.

34. Abbas AK, Lichtman AH and Pober JS: Cellular and Molecular Immunology. WB Saunders, Philadelphia, pp226-242, 1991.

35. Krishnamurthy P, Lambers E, Verma S, et al: Myocardial knockdown of mRNA-stabilizing protein HuR attenuates post-MI inflammatory response and left ventricular dysfunction in IL-10-null mice. FASEB J 24: 2484-2494, 2010.

36. Krown KA, Page MT, Nguyen C, et al: Tumor necrosis factor alpha-induced apoptosis in cardiac myocytes: involvement of the sphingolipid signaling cascade in cardiac cell death. J Clin Invest 98: 2854-2865, 1996.

37. Filippo CD, Cuzzocrea S, Rossi F, et al: Oxidative stress as the leading cause of acute myocardial infarction in diabetics. Cardiovasc Drug Rev 24: 77-87, 2006.

38. Davis ME, Seshadri G, Dikalov S, et al: Delivery of SOD with polyketal particles protects rats from acute myocardial infarction. Circulation 120: S747, 2009.

39. Zheng W, Huang LZ and Zhao L: Superoxide dismutase activity and malondialdehyde level in plasma and morphological evaluation of acute severe hemorrhagic shock in rats. Am J Emerg Med 26: 54-58, 2008

40. Gerster $\mathrm{H}$ : $\beta$-Carotene, vitamin $\mathrm{E}$ and vitamin $\mathrm{C}$ in different stages of experimental carcinogenesis. Eur J Clin Nutr 49: $155-168,1995$.

41. Konukoglu D, Serin O, Kemerli DG, et al: A study on the carotid artery intima-media thickness and its association with lipid peroxidation. Clin Chim Acta 277: 91-98, 1998. 